\section{Drug safety}

There is a pressing need to improve the understanding of safety issues during drug development and post-approval. Our interviewees this month explain their roles in efforts to enhance drug safety.

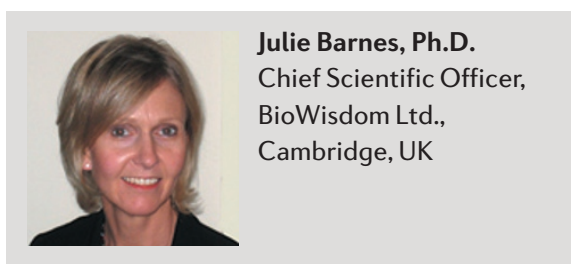

Recent high-profile issues with drug safety have highlighted the need not only to improve post-marketing pharmacovigilance but also to identify drug candidates with good safety profiles earlier. To address these challenges, healthcare technology companies such as BioWisdom are providing intelligence to support decisionmaking about safety issues that arise during the development of new drugs. "Our approach is founded on the principles of ontology. This is a traditional science that applies a relationshipbased approach to, for example, understand mechanisms of drug action as they pertain to adverse events," explains CSO Julie Barnes.

Barnes' career in drug development began when, following her Ph.D. in behavioural neuroscience, she took a postdoctoral position at GlaxoSmithKline (GSK) to develop a series

Craig Hartford,
M.B.B.Ch., M.Sc. Med.,
Ph.D. Executive
Director and Therapeutic
Area Group Head in
Drug Safety and Risk

Traditionally, pharmacovigilance may have been seen as largely drug adverse-event case processing, relatedness assessment and regulatory compliance reporting. However, recent environment changes inside and outside the pharmaceutical industry have resulted in benefit-risk management now being placed in the centre of drug development and drug lifecycle maintenance. "It brings together several medical scientific disciplines such as safetydata processing, safety-signal detection (data mining), assessments of preclinical and clinical potential safety concerns/risks, enhancing safety monitoring, communication of identified safety risks and providing strategies for and implementing risk minimization, to optimize the benefit-risk of medicines," says Craig Hartford, Therapeutic Area Group Head in Drug Safety and Risk Management at Pfizer. of in vivo models and screens for evaluating new chemical entities for Alzheimer's disease. Although initially very specialized in her discipline, over the next 15 years, she began to recognize the value of multidisciplinary research to bring different perspectives and technologies to a business problem.

"While at GSK, I saw projects come and go, clinical trials succeed and fail, and markets change," she says. "Managing change and decision-making in such an environment is an experience that I am pleased to have had the opportunity to acquire."

During this time, Barnes also experienced the rapid expansion in electronic information, which changed the behaviour of scientists from browsing current journals in company libraries to searching e-publications and other electronic data from their desktops. "Getting access to relevant information without being deluged with too much became an increasing challenge," she explains.

BioWisdom was founded on a vision that recognized the need to bring information together in a way that facilitated deeper meaning and interpretation of isolated data points. "There was a 'meeting of minds'
Hartford's interest in drug safety began while he was completing his Ph.D. at the University of Witwatersrand in Johannesburg, South Africa. During this time, he was an investigator for several clinical studies, giving him the opportunity to interact directly with the pharmaceutical industry. This provided an insight into drug and medical-device research, showing that the industry offered a career path for both medical scientists and clinicians. Hartford could see that the pharmacovigilance/benefit-risk physician is typically a core member of the drug development teams. "Collaboration with investigators and researchers, regulators, ethics bodies, practising healthcare professionals and patient groups becomes commonplace for persons working in pharmacovigilance and safety risk management," he explains.

Hartford considers that it is this collaborative and inclusive approach — for example, by always consulting several subject-matter experts when conducting projects - that is vital to success. Describing the importance of this he says: "the sharing of knowledge itself is key to innovation and productivity within pharmaceutical R\&D, and industry is typically very good at encouraging such sharing amongst its many researchers."

Sharing information, although essential given that most new medicines continue

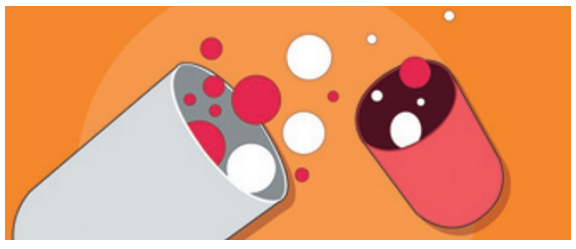

between BioWisdom's goal and my late-stage experience in the industry," says Barnes.

She joined BioWisdom when it was a small start-up; now a rapidly growing business, BioWisdom works with most of the major pharmaceutical companies. "Building strong relationships with like-minded people is very rewarding," she continues, "I have the opportunity to collaborate with scientific leaders and influence how they can make more informed decisions to pursue the best opportunities."

Although Barnes attributes her ability to relate to pharmaceutical partners to her 15 years at GSK, it is her 7 years' experience at BioWisdom that taught her the persistence necessary to establish and grow a new company. "Running a small innovative company is very challenging, requiring tenacity, strength of character, confidence in one's convictions and an ability to persuade people to change what they do to something better. It's really about finding the early adopters, supportive evangelists who can then work with you to validate the approach and spread the word." She concludes: "many small companies fail because they don't realize that success is just around the corner." to be discovered and developed largely within the private sector, is not without its challenges. "Providing organized, planned and proactive methods for clinical safety data review is increasingly important to be able to interpret and act on this data," says Hartford. Technological advances have facilitated the collection of large amounts of drug-safety data, but getting the most valuable information from this and interpreting it correctly to ensure that medicines reach the people who will benefit, both during drug development and following approval, remains an ongoing and important pursuit. "There have been major changes in — and there are continued opportunities for optimizing the way drug-safety data is handled across the drug life-cycle. It is very rewarding to help drive the cultural changes both inside and outside the pharmaceutical industry to focus on the benefit-risk management of medicines," explains Hartford. "The ultimate reward for me, however, is in providing patients with unmet medical needs access to innovative medicines."

\section{WEB SITE}

For more Career snapshots, please see:

Career snapshots: http://www.nature.com/naturejobs/ magazine/career_snaps.html 